\title{
América Latina en el currículo chileno de Historia: análisis desde la literacidad crítica
}

Latin America in the Chilean History

Curriculum: Analysis from Critical Literacy Perspective

A América Latina no currículo de história do Chile: análise numa perspectiva de alfabetização crítica

Jesús Marolla-Gajardo* iD orcid.org/0000-0001-6215-0010 Camila Saavedra-Solís** iD orcid.org/0000-0002-3285-1059

Para citar este artículo: Marolla-Gajardo, J. y Saavedra Solís, C. (2021). América Latina en el currículo chileno de Historia: análisis desde la literacidad crítica. Revista Colombiana de Educación, l(82), 61-82. https:// doi.org/10.17227/rce.num82-10499

\begin{tabular}{lr}
\hline (c) $\bigcup_{\text {BY }} \$$ & Recibido: $16 / 09 / 2019$ \\
$17 / 06 / 2020$
\end{tabular}

* Doctor en Educación en Didáctica de las Ciencias Sociales por la Universidad Autónoma de Barcelona (UAB) y profesor de Historia, Geografía y Educación Cívica por la Universidad Metropolitana de Ciencias de la Educación de Chile (uMCE). Correo: jesus.marolla@umce.cl

** Profesora de Historia, Geografía y Educación Cívica. Universidad Metropolitana de Ciencias de la Educación (UMCE). Académica, Universidad Metropolitana de Ciencias de la Educación, Chile. Correo: saavedracami@gmail.com 


\section{Resumen}

El presente artículo de investigación tiene por objetivo analizar el discurso escrito de América Latina en el currículum escolar y en los textos de estudio de Ciencias Sociales chilenos. Los materiales que se utilizaron para tal análisis son aquellos creados desde el año 2009, hasta el año 2015, periodo de continuidad ministerial en cuanto programas y libros de estudio. Se indagó en los contenidos de los programas, tanto en la recurrencia con que se emplea el término América y América Latina, como desde el lugar que ocupa su discurso en la jerarquización de los contenidos y perspectivas con que se hace presente. La metodología empleada fue el análisis documental, sometiendo a análisis tales datos bajo la Literacidad Crítica y sus concepciones. Entre los principales resultados, encontramos que el contenido referido a América Latina se encuentra desfavorecido en la jerarquización curricular frente a relatos centrados en una historia nacional chilena que fomenta los valores patrióticos y la identidad nacional mestiza, y otros contenidos desde la historia universal, la cual, en rigor, tiene relación con los procesos de los países poderosos occidentales desde aspectos bélicos y económicos, primordialmente. Por lo anterior, entre las principales conclusiones conviene destacar la relevancia de trabajar con los estudiantes los programas oficiales pero desde una perspectiva crítica y peligrosa (Giroux, 1998). Además de fomentar una formación del profesorado que permita la crítica y la reforma a los programas oficiales como el planteamiento de materiales que reconozcan la diversidad.

\section{Palabras clave}

Historia de Latinoamérica currículum; textos y multiculturalidad; programas interculturales

\section{Keywords}

Latin America History; curriculum; multicultural textbooks; intercultural programs

\begin{abstract}
The objective of this research is to analyse the written discourse of Latin America as a concept, in the school curriculum within the Chilean Social Sciences study texts. The materials that will be used for this analysis are those produced in the period between 2009 and 2015. This is a period of ministerial continuity in terms of programs and study books. Now, this study investigates the programs' contents, facing the recurrence with which the terms 'America' and 'Latin America' are used. At the same time, this text wants to investigate which places those concepts occupy in the hierarchical organization of contents and perspectives presented. The methodology used was a documentary analysis that gathered data submitted to analysis under Critical Literature and its conceptions. Among the main results, we find that the content referring to Latin America is in a disadvantage in the curricular hierarchical organization compared to other stories focused on a Chilean national history that foster patriotic values and mestizo national identity, where other contents from universal history are primarily related to the processes of the powerful western countries from military and economic aspects. Therefore, among the main conclusions, it is worth highlighting the relevance of working with the official programs and with students but from a critical and dangerous perspective (Giroux, 1998),moreover, to promote teacher training that allows criticism and reform of official programs such as the provision of
\end{abstract} materials that include diversity.

\section{Resumo}

Este artigo de pesquisa pretende analisar o discurso sobre a América Latina no curriculum escolar e nos livros didáticos de Ciências Sociais chilenos. Os materiais que se utilizaram foram criados entre 2009 e 2015 , período de continuidade ministerial enquanto programas e livros de estudo. Indaga-se nos conteúdos dos programas, tanto a recorrência com que se usam os termos América e América Latina, quanto o lugar que ocupa seu discurso na hierarquização dos conteúdos e perspectivas atuais. A metodologia utilizada foram as análises documentais e a submissão dos dados à Alfabetização Crítica e suas concepções. Entre os principais resultados, encontramos que o conteúdo referente à América Latina se encontra desfavorecido na hierarquização curricular frente a relatos centrados na história nacional chilena que fomenta valores patrióticos e a identidade nacional mestiça, e outros conteúdos, desde a história universal, a qual, em rigor, tem relação com os processos dos poderosos países ocidentais, primordialmente assuntos N. ${ }^{\circ} 82$ bélicos e econômicos. Entre as principais conclusões, destaca-se a relevância de trabalhar os programas oficiais com os estudantes, mas desde uma perspectiva crítica (Giroux, 2018). Também de fomentar uma formação do professorado que que reconheçam a diversidade.

\section{Palavras-chave}

História da América Latina; currículo; livros didáticos e multiculturalismo; programas interculturais 


\section{Introducción}

La presente investigación analiza el discurso escrito de América Latina en el currículum escolar y los textos de estudio de Ciencias Sociales chilenos, para el ajuste curricular del periodo 2009-2015'. Los dispositivos curriculares examinados presentan una producción discursiva (Foucault, 1979) donde se tensionan las tradiciones curriculares nacionales frente a la enseñanza de la historia y las ciencias sociales.

Al indagar en los contenidos del subsector de Historia, Geografía y Ciencias Sociales se evidencia que los niveles de segundo, tercero y cuarto año de secundaria se articulan desde la historia nacional, mientras que en primer año de enseñanza secundaria se sostiene una historia en clave universal ${ }^{2}$. Al respecto, Castro (2014) sostiene que las distintas migraciones que han ocurrido en los países de América Latina y el Caribe han promovido la tensión entre los distintos discursos que persiguen construir identidades homogéneas; el caso chileno tiene un claro interés performativo en cuanto construcción social de la población.

Consideramos, siguiendo a (Apple, 1991), que el currículo como dispositivo obedece a los lineamientos hegemónicos que persiguen la formación de identidades y los valores patrios que definen las estructuras sociales con poder. Distintos estudios, como los de Marolla (2014, 2019a, 2019b), Marolla y Pagès (2018); Pagès (1999); Pagès y Mariotto (2014); Pagès y Sant, (2012) y Pinochet $(2014,2015)$ afirman que el currículo y los materiales se limitan a reflejar un concepto que referencia a la masculinidad como estructura social dominante, por sobre la otredad y las diversidades que existen en la sociedad (Marolla, 2019a y 2019b).

Dadas las perspectivas y los problemas educativos actuales en torno a las desigualdades sociales por razones de género, etnia y clase, así como los procesos de interculturalidad y migración a que se asiste como sociedad chilena, consideramos que es fundamental someter a reflexión y análisis los contenidos que transmiten los programas oficiales. Desde nuestras propias prácticas educativas, nos vemos enfrentados a tener que trabajar los procesos de enseñanza y aprendizaje en contextos diversos y desde una lógica ministerial oficial basada en programas que fomentan la homogeneidad y la desigualdad. De ahí que sea de interés educativo y social analizar los contenidos de tales programas a partir de las estructuras discursivas sobre América Latina.

1 Se escoge el marco temporal debido a los ajustes en los programas de Historia, Geografía y Ciencias Sociales introducidos en el 2009, y el anuncio de nuevas modificaciones en el 2015, que finalmente se materializaron para el segundo ciclo básico en el 2016, y para la Educación Media en el 2017.

2 Se entiende la referencia a la "historia universal" ligada, eminentemente, a la historia del Occidente europeo. 


\section{América Latina desde una conceptualización histórica}

La voz América Latina representa, desde su creación por Bilbao y Torres Caicedo, el deslinde histórico-cultural entre Norteamérica y los territorios ubicados al sur del Río Grande hasta Tierra del Fuego. La creación del concepto aspira a frenar el avance de la hegemonía de los Estados Unidos sobre la política latinoamericana y se eleva en resistencia a la amenaza del intervencionismo (Zea, 1971).

Mignolo (2007) identifica que los procesos independentistas marcan el reordenamiento del mundo moderno/colonial y deben entenderse en relación con un imperialismo en ascenso en Europa y Estados Unidos, "apoyado por una economía capitalista y en el deseo de determinar la forma de la emancipación del mundo no europeo" (p. 79). Desde las independencias hasta fines del siglo xIx, el continente enfrenta la necesidad de afirmar la propia identidad, mientras resiste la embestida de las potencias imperialistas de la época (Arpini, 2013).

Desde el periodo de las independencias hasta fines del siglo xIx, el continente se debatió entre las corrientes que planteaban la construcción de una identidad latinoamericana y las imposiciones de las potencias imperialistas. Sin embargo, para Arpini (2013), aspectos como la autoafirmación, el autorreconocimiento y la unidad político-cultural, quedan relegados bajo las definiciones y la continuidad de las jerarquías coloniales (Marolla y Saavedra, 2020).

La filosofía latinoamericana y el latinoamericanismo se forjaron desde el siglo xvı y hacia los procesos independentistas se destacaron las obras de pensadores como Francisco Miranda, Simón Bolívar, Juan Bautista Alberdi y José Enrique Rodó, entre otros. Tal como señala Saladino, la lucha de los latinoamericanos "interesados en fundamentar la identidad y ser del latinoamericano y de América Latina" (2010, p. 180) es un desafío histórico y una refutación al colonialismo intelectual que desde la conquista española perpetuó el descrédito a las culturas originarias, y luego, a la producción cultural e intelectual de criollos y mestizos americanos.

Saladino (2010) sostiene que el latinoamericanismo, como pensamiento descolonizador, promueve la relectura del pensamiento y la historia de las ideas latinoamericanas, articulándose desde el desarrollo histórico de una crítica al colonialismo, la conceptualización de América Latina como espacio soberano y Latinoamérica como expresión de su historia y espacio de desarrollo cultural. 
Asimismo, Roig (1993) sostiene que la historia de las ideas latinoamericanas recompone el camino zigzagueante del sujeto latinoamericano, y se articula según Zea (1971) como una actividad que, lejos de tener un interés erudito, se instala desde una herramienta para el autoconocimiento del ser latinoamericano, a la vez que articula "la continuidad de voces que de época en época van mostrando el ejercicio de la subjetividad del hombre latinoamericano" (Roig, 1993, p. 165).

De esta manera, se articula y construye América Latina como objeto de estudio que representa la lucha histórica de afirmación y reconocimiento de las sociedades latinoamericanas, en especial desde la lucha contrahegemónica frente a las narrativas coloniales que son parte de la historiografía tradicional.

Lo anterior se podría traducir a la luz de las problemáticas históricas en el contexto de producciones discursivas de poder (Foucault, 1979). La historia de América Latina, por tanto, viene a ser una historia de luchas de poder en torno a la construcción de discursos que la definen desde el discurso y rol en el sistema-mundo y dependencias económicas, políticas y culturales (Castro, 2014; Saladino, 2010; Tubino, 2005).

Para el escrito, nos posicionamos en una visión desde los márgenes y el abismo (Santos, 2011) sobre América Latina como concepto y discurso (Quijano, 2000). Consideramos la perspectiva de las epistemologías del sur (Santos, 2011; 2017), comprendidas como los nuevos procesos de producción y de valoración de los nuevos conocimientos desde los grupos tradicional y socialmente excluidos por el capitalismo y el colonialismo (Tamayo, 1975). Esta aproximación teórica (Santos, 2017) es, precisamente, la base de nuestro análisis de los programas ministeriales.

La estructura de la colonialidad se extiende a la historiografía latinoamericana al ser silenciada y cifrada como fragmentados marginales de la historia oficial, erguida como relatos incompletos y rupturas históricas sin significado (Fernández, 2012). La modificación de los patrones coloniales que imperan en la construcción del conocimiento histórico supone desplegar prácticas que cuestionen la narrativa colonial trazada por el colonizador y sean reemplazadas por narrativas articuladas desde la perspectiva del colonizado (Santos, 2009). En esta línea, la perspectiva decolonial sostiene que existe una relación entre poder y saber históricamente construida, que determina que el colonialismo se haya extendido globalmente. La colonialidad se concibe como una red de relaciones raciales, étnicas, epistémicas, control hegemónico e imposición de conocimientos, arraigadas en las sociedades colonizadas (Quijano, 2000; Santos, 2009). 


\section{La comprensión de los materiales curriculares desde la teoría crítica}

El concepto de currículo y programas ha sido ampliamente tratado desde la teoría crítica (Apple, 1991; Apple y Beane, 2012; Giroux, 1990; 1998; Pinar, 2014). Para este estudio, consideramos que el currículo es una construcción hegemónica que obedece a estructuras de poder emanadas tanto desde posiciones jerárquicas de los Gobiernos como desde aquellas estructuras que monopolizan los poderes políticos y económicos (Apple, 1998; Pinar, 2014). Así, como dicen Apple y Beane (2012), el currículo funciona como una expresión que pretende transmitir cultura. Sin embargo, no es cultura en el sentido amplio de la palabra, sino que es la cultura que las estructuras hegemónicas quieren transmitir y socializar hacia la población (Marolla y Saavedra, 2020).

Apple dice que el currículo se encarga de la "distribución de las normas y disposiciones convenientes al lugar que ha de ocupar cada uno en una sociedad jerárquica" (1991, p. 33), dejando al margen las posibilidades de desarrollo del pensamiento crítico y la reflexión sobre las problemáticas sociales. Incluso, el currículo no solo es la expresión de las normas y de los valores de la cultura dominante, sino que se encarga de silenciar las voces, los problemas y las historias de aquellos que se encuentran fuera de las estructuras de poder, tales como las personas de color, las etnias, las mujeres y los jóvenes (Apple y Beane, 2012).

Giroux (1998) afirma que los currículos emanados desde las estructuras de poder pasan a formar parte de la tradicionalidad educativa. En ese sentido, emerge un tipo de formación que no propone la reflexión sobre el tipo de conocimiento que se quiere enseñar en las escuelas. Tampoco se cuestionan las formas en que ha construido el conocimiento histórico, ni la historia propuesta para enseñar: "La escuela acalla activamente a los estudiantes a través de ignorar sus historias, de encuadrarlos dentro de clases con expectativas mínimas y de negarles conocimientos relevantes para ellos" (Giroux, 1998, pp. 80-81). De ahí que no exista una reflexión sobre cómo se construyen los distintos tipos de conocimiento (Apple y Beane, 2012; Giroux, 1998).

El currículo es un instrumento ideológico que busca el mantenimiento del statu quo social, en el cual los Gobiernos y aquellos que controlan las estructuras de poder entregan un conocimiento y una visión de la realidad y la historia que no alteran el orden existente; al contrario, reafirman las estructuras y sus posiciones de poder y privilegios (Barton, 2002). Si se somete a análisis el contenido que guía el currículo, así como las finalidades que persigue, se podrá vislumbrar las formas en que se han construido la historia, la sociedad y las estructuras de poder (Bonilla y Martínez, 1992). Cuesta (1998), desde una perspectiva analítica plantea que la historia escolar 
se compone de tres niveles o capas: la historia regulada, entendida como aquella que la administración educativa reconoce como historia valiosa, que compone los programas y los textos escolares; la historia soñada, articulada desde "grupos innovadores" que, frente a la enseñanza tradicional, desarrollan propuestas transformadoras; y, finalmente, la historia enseñada, que es la que efectivamente se imparte en la sala de clases.

Desde las perspectivas críticas, nos basamos en la teoría poscolonial (Santos, 2014; 2017), que sustenta los discursos desde el indigenismo, así como desde lo intercultural. Estas se entienden como aproximaciones reivindicadoras que persiguen el empoderamiento y la transformación de las desigualdades por razones de etnia, clase y género de aquellas comunidades que han estado marginadas y ausentes en la construcción histórica (Fraser, 2008; Quijano, 2000; Tubino, 2005; Woyshner, 2002). Lo anterior, refiere Quijano (2000), se define como la "colonialidad del poder"; tales referencias hacen alusión a las estructuras que se han construido desde escalas de identidades sociales con el blanco europeo en las jerarquías superiores, y que han dejado a los aborígenes y a los afrodescendientes en las jerarquías inferiores (Santos, 2011).

\section{Metodología}

\section{Objetivos del estudio}

El problema de investigación busca indagar, a través del currículo escolar, las representaciones y los discursos escritos en torno a los contenidos y sus protagonistas. El periodo de los documentos comprende los años 2009 y 2015, ya que en el primero se realizó una importante reforma a las bases y programas, en tanto que en 2015 se realizó una segunda reforma que marcó los lineamientos de la educación chilena y la enseñanza de la historia y las ciencias sociales (Ministerio de Educación República de Chile-Mineduc, 2009; 2011 y y 2011b; 2015a y 2015b). Las preguntas que nos planteamos fueron:

¿ ¿Cuáles son las representaciones sobre América Latina en el currículo escolar chileno y en los textos de estudio de Ciencias Sociales?

¿ ¿Cuáles son los discursos bajo los que se incluyen los protagonistas en el currículo de historia, geografía y ciencias sociales?

Para el desarrollo del estudio, se proponen los siguientes objetivos:

"Analizar las representaciones y los contenidos relacionados con América Latina en el currículo chileno (2009-2015).

» Examinar y reflexionar en torno a quiénes protagonizan el currículo de Historia, Geografía y Ciencias Sociales en lo referente al discurso sobre América Latina. 
En cuanto al trabajo metodológico, podemos referenciar dos áreas relevantes: el análisis documental y el análisis desde las herramientas de la literacidad crítica.

La literacidad crítica (LC) nos aportó las herramientas para analizar y reflexionar acerca del contenido y los discursos que transmiten los programas. Como plantean Cassany (2006), Tosar (2018) y Ortega y Pagès (2017), la LC nos brinda útiles herramientas que nos permiten comprender ideológicamente los discursos históricos, tanto en la lectura como la escritura, y analizar los discursos con una mirada crítica. De esta manera, la Lc colabora en que los estudiantes puedan leer las líneas (una comprensión literal), leer entre líneas (comprensión inferencial) y leer tras las líneas (comprensión de la ideología) (Santisteban, 2015). Por ello, siguiendo a Tosar (2018, p. 7), las preguntas metodológicas desde la LC que nos guían son:

» ¿Quién está representado? ¿Qué voces faltan?

¿QQuién se beneficia de esto?

¿QQué medidas podrías tomar con base en el texto?

Consideramos esencial el uso de las referencias desde la LC, ya que nos permite repensar y reflexionar más allá del propio contenido y discurso que nos entregan los materiales. De ahí que podamos visualizar, desde la didáctica de las ciencias sociales, las representaciones, así como el rol que se otorga a quienes protagonizan los programas oficiales del Gobierno.

\section{Contexto del estudio}

El informe "La educación chilena en el cambio de siglo" de la Organización de las Naciones Unidas para la Educación, la Ciencia y la Cultura- Unesco (2004) plantea que la transición a la democracia inaugura un amplio proceso de reforma educacional. Hacia 1996, la reforma educativa contempló la creación del nuevo Currículo Nacional para Educación Primaria y Secundaria. Este se materializó en el decreto 220 de 1998, que estableció los objetivos fundamentales y contenidos mínimos obligatorios para la enseñanza secundaria. El decreto anterior fue fijado por el Ministerio de Educación chileno, creado al alero de la Ley Orgánica Constitucional de Enseñanza (LOCE).

La LOCE dispone que el Ministerio de Educación es el encargado de crear los planes y programas para aquellos establecimientos educacionales que no elaboren los propios. Los estudios desarrollados por Cox (2003) y Bellei (2001) sostienen que más del $75 \%$ de las escuelas del país no han instaurado planes y programas propios, lo que determina que el currículo regulado tenga amplio alcance en la educación nacional (Cuesta, 1998). Un reciente informe de la Unesco (2018) recoge las apreciaciones 
del Colegio de Profesores de Chile (2009), y coincide con Falabella (2015) al plantear que la prescripción curricular y los instrumentos de evaluación nacional

omiten la diversidad cultural y las capacidades de los estudiantes [...] Sancionan y estigmatizan a la educación pública [...] Se aplican de modo homogéneo negando las necesidades educativas de los alumnos/as [...] [y] Promueven la competencia para alcanzar los premios de excelencia académica. (Colegio de Profesores, p. 5)

El currículo prescrito por el Mineduc se organiza a través del Marco curricular y el Programa de estudios. El primero es el documento principal que define los aprendizajes que los estudiantes deberían lograr durante su trayectoria escolar; el segundo, propone la organización didáctica del año escolar por asignatura, está estructurada a partir de los contenidos mínimos obligatorios (CMO), que son los conocimientos, habilidades y actitudes que constituyen los objetivos fundamentales que deben adquirir los estudiantes a lo largo de toda la enseñanza primaria y secundaria.

\section{Resultados}

Los materiales curriculares analizados corresponden al periodo 2009-2015. Dicho septenio se escogió como marco temporal, ya que durante estos años se diseñó e implementó una reforma curricular. En el cuadro 1 se enumeran los materiales analizados.

\section{Cuadro 1.}

Materiales utilizados en el estudio

Ministerio de Educación República de Chile (2009). Objetivos fundamentales y contenidos mínimos obligatorios de la educación básica y media.

Ministerio de Educación República de Chile (20lla). Historia, Geografia y Ciencias Sociales. Programa de estudios primer año medio.

Ministerio de Educación República de Chile (2011b). Historia, Geografía y Ciencias Sociales. Programa de estudios segundo año medio.

Ministerio de Educación República de Chile (2015a). Historia, Geografía y Ciencias Sociales. Programa de estudios tercer año medio

Ministerio de Educación República de Chile (2015b). Historia, Geografía y Ciencias Sociales. Programa de estudios cuarto año medio.

Fuente: elaboración propia

Desde la LC, se hace necesario cuantificar algunos aspectos sobre la presencia de América Latina como contenido en los programas antes mencionados. Así, se seleccionaron aquellos смо que presentaban entre 
sus descriptores a América/América Latina de un modo directo y explícito. Consideramos los planteamientos de Tosar (2018), siguiendo las preguntas de ¿quién está representado?; ¿qué voces faltan?; ¿quién se beneficia de esto?; ¿qué medidas podrías tomar con base en el texto ${ }^{3}$ La información se presenta en la tabla 1, donde en cada nivel existen siete $\mathrm{cmO}$, con excepción de segundo medio, donde se encuentran ocho.

\section{¿Quién está representado?}

En la tabla 1 se identifica que, de una totalidad de 29 смо en los programas ministeriales, solo hay ocho que aluden directa o indirectamente al concepto de América Latina. En cuanto a porcentajes, desde primero hasta cuarto año de enseñanza secundaria, la presencia de los cmo sobre América Latina es de un $27 \%$ con relación al total de contenidos incluidos en los documentos ministeriales. No obstante, esto no quiere decir que un $27 \%$ de los смо se dediquen a América Latina de manera profunda y exhaustiva, sino que el concepto aparece al menos nombrado, referido o señalado.

Tabla 1.

Contenidos mínimos obligatorios asociados a América Latina

\begin{tabular}{lccc}
\hline Nivel (año) & $\begin{array}{c}\text { cmo asociados a } \\
\text { América Latina }\end{array}$ & Total cmo & $\begin{array}{c}\text { Porcentaje del } \\
\text { total (\%) }\end{array}$ \\
\hline Primero & 4 & 7 & 57 \\
\hline Segundo & 2 & 8 & 25 \\
\hline Tercero & 1 & 7 & 14 \\
\hline Cuarto & 1 & 7 & 14 \\
\hline & 8 & 29 & 27 \\
\hline
\end{tabular}

Fuente: elaboración propia

En cuanto al diagnóstico de la centralidad del concepto, o bien, estableciendo si se trata de un concepto descentrado que se subordina a otras temáticas (Miralles y Gómez, 2017), se identifican dos subcategorías que corresponden a la presencia de América vinculada al tratamiento de temáticas relativas: Estados Unidos y Europa; y Chile y América Latina.

Del análisis de la tabla 2 se infiere que existen dos tendencias que agrupan a la mayoría de los CMO: el 50 \% corresponde a la jerarquización del contenido que da centralidad a Estados Unidos y Europa, siendo América un subtema dentro de las unidades de aprendizaje. El otro 50 \% gira en torno a

3 Las dos primeras preguntas las desarrollaremos a fondo en el apartado de resultados, y dejaremos las dos últimas para el de conclusiones, sin el perjuicio de que se incluyan todos los aspectos de forma breve para fines de análisis en las dos últimas secciones del texto. 
Chile como temática principal, y América se vincula como subunidad. Esto revela que las perspectivas de Chile en el contexto americano están disminuidas frente a la importancia que se da a las perspectivas eurocéntricas del currículo del país (Apple, 1991; Giroux, 2003). Lo anterior guarda relación con la construcción hegemónica de los programas, estableciendo jerarquías en cuanto a los contenidos que se trabajan y la formación de identidades nacionales occidentales (Marolla y Saavedra, 2020).

Tabla 2.

¿Qué lugar ocupa América en la jerarquización de contenido?

\begin{tabular}{lcccc}
\hline \multicolumn{1}{c}{ Nivel } & $\begin{array}{c}\text { Estados Unidos } \\
\text { y Europa }\end{array}$ & Chile y América & Total & $\begin{array}{c}\text { Porcentaje } \\
\text { por nivel (\%) }\end{array}$ \\
\hline $\begin{array}{l}\text { Primero de } \\
\text { secundaria }\end{array}$ & $\begin{array}{c}\mathrm{cmo} \\
\mathrm{cmo} \mathrm{3}\end{array}$ & - & 2 & 33,3 \\
\hline $\begin{array}{l}\text { Segundo de } \\
\text { secundaria }\end{array}$ & - & $\mathrm{cmo} \mathrm{3}$ & 1 & 16,6 \\
\hline Tercero de secundaria & $\mathrm{cmo} \mathrm{3}$ & $\mathrm{cmo} \mathrm{4}$ & 1 & 33,3 \\
\hline Cuarto de secundaria & 3 & $\mathrm{cmo} \mathrm{4}$ & 1 & 16,6 \\
\hline Total cmo & 50 & 3 & 6 & 100 \\
\hline Total \% cmo & 50 & & 100 \\
\hline
\end{tabular}

Fuente: elaboración propia

Los porcentajes obtenidos permiten aseverar que, aunque existen 7 смо que hacen referencia al tema de América y algunos aspectos relacionados, solo en un contenido el concepto actúa como temática central, mientras que en el 85,6 \% de los casos aparece como subtema vinculado a otros contenidos. Por ello, la representación que se entrega sobre América y América Latina desde su diversidad obedece a aspectos subsidiarios y subordinados a otras referencias y contenidos de la historia y las ciencias sociales (Spivak, 2012).

Todo lo anterior se enmarca en las perspectivas que buscan construir una imagen de Chile en el contexto americano como un país occidental, ligado a Europa y su cultura, siendo por ello blanco, homogéneo y con alta presencia masculina. Se podría definir como una perspectiva funcional a la historia dominante, donde la multiculturalidad o la interculturalidad se trabajan desde una visión que no somete a reflexión ni crítica las estructuras sociales (Walsh, 2012). Es decir, se proponen el diálogo, la convivencia, la tolerancia y la inclusión, pero todas ellas en función de las estructuras de poder y las lógicas del modelo actual político y económico. La primera subcategoría de análisis es América como subtema vinculado a Estados Unidos y Europa. En el cuadro 2, se muestra el desglose de los cmo referidos. 


\section{Cuadro 2.}

América como subtema vinculado a Estados Unidos y Europa

\begin{tabular}{|c|c|}
\hline Смо & Descriptor смо \\
\hline $\begin{array}{l}\text { сMO } 1 . \\
\text { Primer año }\end{array}$ & $\begin{array}{l}\text { Análisis del impacto económico y social, en Europa y } \\
\text { América, de la gran crisis de la economía capitalista. } \\
\text { (Primer año de secundaria, 2011, p. 92) }\end{array}$ \\
\hline \multirow{2}{*}{$\begin{array}{l}\text { сMO } 3 . \\
\text { Primer año. }\end{array}$} & $\begin{array}{l}\text { Identificación de nuevos actores en el escenario mundial en } \\
\text { las dinámicas de la Guerra Fría: procesos de descolonización, } \\
\text { desarrollo de movimientos revolucionarios en América } \\
\text { Latina. (Primer año de secundaria, 20ll, p. 92) }\end{array}$ \\
\hline & $\begin{array}{l}\text { Descripción de los principales procesos económicos y } \\
\text { políticos de fines de siglo: crisis del Estado de Bienestar e } \\
\text { implementación de políticas neoliberales; terrorismo de Estado } \\
\text { y violación de los derechos humanos en América Latina y } \\
\text { en otras regiones. (Primer año de secundaria, 2011, p. 92) }\end{array}$ \\
\hline \multirow{2}{*}{$\begin{array}{l}\text { смо } 3 . \\
\text { Tercer año }\end{array}$} & $\begin{array}{l}\text { Identificación de América Latina como zona de influencia } \\
\text { norteamericana durante la Guerra Fría. (Tercer año medio, 2015, p. 14) }\end{array}$ \\
\hline & $\begin{array}{l}\text { La política norteamericana hacia América Latina } \\
\text { (Alianza para el Progreso y doctrina de seguridad } \\
\text { nacional). (Tercer año medio, 2015, p. 14) }\end{array}$ \\
\hline
\end{tabular}

Fuente: elaboración propia

En el смо 3 de primer año de enseñanza secundaria se afirma que el proceso histórico central es la Guerra Fría desde una perspectiva historiográfica. Los nuevos actores en el escenario mundial no tienen relevancia ni pertinencia para América Latina, sino que los discursos, los escenarios y los protagonistas son parte del mundo europeo, blanco, occidental y masculino (Tamayo, 1975). En tal punto, solo se remiten a relatar contenidos ligados a procesos económicos y políticos de finales del siglo, como el Estado de bienestar, las políticas neoliberales, entre otras. En especial se hace mención a las violaciones de los derechos humanos, no obstante, son trabajados desde perspectivas que no cuestionan el proceso. El currículo resalta la descripción de acontecimientos históricos que se relacionan con temas de política y economía, en general, desde la visión oficial del Gobierno (Marolla y Pagès, 2018; Ortega et ál., 2020; Pagès y Sant, 2012).

Lo anterior se puede explicar debido a la naturaleza —en muchas ocasiones- traumática de los procesos que se trabajan. Desde las hegemonías se realiza una selección sobre el pasado que se recordará en función de los intereses del presente y las estructuras de poder (Torres, 2016). Aspectos como la memoria, los protagonistas y los eventos-escenarios se van construyendo y reconstruyendo constantemente de acuerdo con los intereses de los grupos dominantes. Sarlo dice que "la memoria coloniza el pasado y lo organiza sobre la base de las concepciones y las emociones del presente" (2012, p. 92). 


\section{¿Qué voces faltan? América Latina como contenido y discurso homogéneo}

Se puede asumir que la visión de América Latina se ha construido desde la perspectiva establecida por la política internacional, donde prima una lectura del continente basada en la visión global/mundial de los países hegemónicos occidentales. Por ejemplo, los cmo tratan la segunda etapa del siglo xx para América Latina desde una visión que considera la Guerra Fría como el evento/acontecimiento que condiciona el resto de los procesos del continente.

En el cuadro 3 se puede apreciar que América, desde la LC es concebida con relación a contenidos que tienen que ver con la historia de Chile. Esto es interesante, ya que no se resaltan procesos propios sobre la heterogeneidad étnica ni la diversidad propia latinoamericana. En efecto, los procesos vinculados a Chile agrupan un 42,8 \% de los cmo.

\section{Cuadro 3.}

América como subtema vinculado a Chile

\begin{tabular}{|c|c|}
\hline смо & Descriptor смо \\
\hline \multirow{2}{*}{$\begin{array}{l}\text { cmo } 3 . \\
\text { Segundo año } \\
\text { de secundaria }\end{array}$} & $\begin{array}{l}\text { Discusión sobre los múltiples factores que precipitaron el } \\
\text { proceso independentista en América y Chile: la crisis de la } \\
\text { monarquía española; la maduración de las aspiraciones políticas } \\
\text { de las élites criollas; condiciones estructurales y acciones } \\
\text { individuales. (Segundo año de secundaria, 20ll, p. 10l) }\end{array}$ \\
\hline & $\begin{array}{l}\text { Análisis de la guerra de Chile contra la Confederación Perú- } \\
\text { Boliviana como uno de los conflictos bélicos generados } \\
\text { en el proceso de conformación de los nuevos Estados } \\
\text { hispanoamericanos. (Segundo año de secundaria, 2011, p. 101) }\end{array}$ \\
\hline $\begin{array}{l}\text { сMO } 4 . \\
\text { Tercer año de } \\
\text { secundaria }\end{array}$ & $\begin{array}{l}\text { Contextualización del proceso político chileno en el marco } \\
\text { de las dictaduras y la recuperación de la democracia en } \\
\text { el Cono Sur. (Tercer año de secundaria, 2015, p. 182) }\end{array}$ \\
\hline $\begin{array}{l}\text { cmo } 4 . \\
\text { Cuarto año de } \\
\text { secundaria }\end{array}$ & $\begin{array}{l}\text { Caracterización de las políticas económicas de Chile para su inserción } \\
\text { en la economía global: la apuesta por desarrollar las exportaciones; } \\
\text { la opción bilateral y la participación en bloques económicos; los } \\
\text { tratados de libre comercio. (Cuarto año de secundaria, 2015, p. 15) }\end{array}$ \\
\hline
\end{tabular}

Fuente: elaboración propia

La articulación del cmo 3 de segundo año de secundaria sugiere que los temas de interés del currículo y que relacionan los acontecimientos de Chile con los de América Latina solo se remiten a los aspectos políticos, económicos y bélicos. Desde la LC, es posible afirmar que se entrega una perspectiva de semejanza, ya que se posicionan en un mismo nivel los temas relacionados con Chile y América. El resto de países que aportan heterogeneidad, y evidencian la diversidad del continente, se omiten. 
En el CMO 4, es posible afirmar, con base en la LC, que se tratan los eventos económicos que han involucrado a Chile en el marco de los procesos de América Latina; se propone una visión positiva y de progreso económico sobre el mercado y el neoliberalismo, con una amplia participación del país en las dinámicas globales. No se problematizan las condiciones de dicho proceso histórico y sus implicancias presentes y futuras.

Los CMO vinculados a Chile y América sugieren, desde la misma perspectiva de análisis, que el discurso articulado en el currículo presenta una relación discontinua temporal entre Chile y América. Los CMO se centran en los procesos de las guerras de Independencia, el periodo posindependentista, la Guerra Fría y el Chile actual. Aspectos como las guerras de Independencia o la diversidad propia del proceso llevado a cabo por los territorios latinoamericanos son omitidos, en las prácticas del profesorado y a pesar de su presencia en el currículo, en función de dar relevancia a la influencia de la Independencia de los Estados Unidos y la Revolución francesa como ejes articuladores del proceso (Falabella, 2015; Tamayo, 1975).

La existencia de solo tres contenidos mínimos que aludan al caso y los procesos relativos hacia América Latina demuestra que la conceptualización histórica que prima es la del particularismo histórico de Chile y la homogeneidad en cuanto a protagonistas, políticas y escenarios por encima de otras visiones históricas que apuestan por la integración latinoamericana, así como por la diversidad y las distintas visiones sobre los acontecimientos históricos.

\section{Conclusiones}

\section{¿Quién se beneficia de esto? ¿Hacia dónde apunta el currículo chileno?}

La observación de la jerarquización temática para la enseñanza de la historia, la geografía y las ciencias sociales, permite afirmar que la historia enseñada prioriza la historia universal europea en primer año de secundaria y la historia nacional en segundo, tercero y cuarto año de enseñanza para el mismo nivel. Así, la enseñanza de América Latina se supedita a procesos históricos centrales con foco en los Estados Unidos, Europa y las relaciones de tales hegemonías con un Chile subordinado (Arpini, 2013; Tamayo, 1975).

Entre América Latina, los Estados Unidos y Europa es posible establecer una estructura discursiva (Foucault, 1979) que hace primar las relaciones asimétricas de poder, en la medida en que se representa a las potencias europeas y norteamericanas como rectoras de las relaciones internacionales. Latinoamérica se figura como un escenario pasivo en la geopolítica 
mundial, zona de influencia depositaria de las fuerzas históricas surgidas de los Estados Unidos. De esta manera, mientras el Norte es articulado como gestor de las relaciones continentales, América Latina se conceptualiza como espacio de subordinación incapaz de frenar la política imperialista de los Estados Unidos (Quijano, 2000; Spivak, 2012). Esto se puede tomar como la "colonialidad del poder", donde los Estados Unidos conduce los procesos, y tanto Chile como América Latina están sujetas a las jerarquías ideológicas establecidas.

Referente a la correlación entre la historia de Chile y América Latina, como contenidos, espacios y discursos de poder desde y para la enseñanza, estos se abordan desde un enfoque histórico que privilegia la generación de una historia excepcional y unilateral, focalizando los discursos desde la diferencia con los países vecinos y el escenario latinoamericano (Quijano, 2000; Tubino, 2005), y se omiten tanto la historia en común, como la propia diversidad del continente.

Es posible plantear que la estructura discursiva (Foucault, 1979) en torno a los contenidos de América Latina figura las tensiones conceptuales que grafican diversas perspectivas de poder, las cuales se abordan en el currículo escolar. Los conceptos geográficos que indican territorios o continentes no son términos técnicos neutros, sino que constituyen conceptos ideológicos y políticos que fijan quién pertenece y quién está fuera de una comunidad (Foucault, 1979; Spivak, 2012; Tubino, 2005). América Latina es conceptualizada en torno a la oposición y diferencia respecto de los Estados Unidos, y pasa a ser un continente homogéneo y contraparte en una jerarquía inferior a la hegemonía del país del Norte. Se omite la diversidad multicultural, así como las distintas perspectivas y procesos que han ocurrido en el continente y que han sido un aporte para la construcción de los distintos territorios (Marolla, 2014; 2019a; Pagès y Marolla, 2018).

Para contribuir a la formación y a las prácticas del profesorado, es necesario problematizar la forma en que se comprenden los contenidos, así como los discursos según los cuales se problematizan los distintos tipos de conocimientos (Barton, 2002; Castro, 2014; Pagès y Santisteban, 2015). Es una manera de comprender los procesos de exclusión simbólica que se manifiestan (Plá, 2016; Pagès y Marolla, 2019b). La transformación curricular debe considerar la historia soñada y la decolonialidad en torno al conflicto, de manera que se identifiquen los focos problemáticos — como la xenofobia, el racismo y los nacionalismos-, a fin de abordar en la práctica los problemas sociales vivos, y todo discurso de odio que promueva la desigualdad y las violencias (Santisteban, 2015).

A pesar de que la formación del profesorado (Barton, 2002; Bonilla y Martínez, 1992; Castro, 2014; Marolla, 2019b) tenga propósitos críticos para la transformación de las desigualdades sociales y la invisibilidad de 
las diversidades propias de las comunidades, es presa de un currículo prescriptivo que responde a los lineamientos hegemónicos de los grupos en las jerarquías de poder (Ortega y Pagés, 2017; Plá, 2016; Giroux, 2003). De ahí que se tenga que plantear, tanto desde las prácticas del profesorado como desde los programas, una reforma radical con perspectiva crítica (Walsh, 2012) y una historia soñada (Cuesta, 1998), a fin de proponer un currículo y unas prácticas enfocadas en la lucha contra los prejuicios, los estereotipos y la invisibilidad, para dar agencia a los espacios de empoderamiento y lucha por la justicia social de la diversidad de género, de etnia y de clase ante aquellas estructuras que las han marginado.

\section{¿Qué medidas se podrían tomar con base en los programas para la formación del profesorado?}

Para las prácticas y la reflexión en torno al trabajo del profesorado, se coincide en que la noción de diferencia debe ser replanteada, considerándola positivamente en función de comprender a la otredad (Walsh, 2012; Pagès y Sant, 2012). Esto requiere de una historia latinoamericana que configure relatos históricos que visibilicen la diferencia, ya sea desde el ámbito cultural entre sujetos históricos, o desde una historia comparada que permita establecer contrastes entre las historias nacionales latinoamericanas (Quijano, 2000).

Con base en el análisis efectuado, podría definirse junto con Cuesta (1998) que el currículo, los programas y el discurso de América Latina se enmarcan en las perspectivas de historia regulada, donde las administraciones educativas reconocen como el único conocimiento válido, el contenido en los programas y el currículo oficial. De ahí que la propuesta del escrito, considerando perspectivas decoloniales (Santos, 2017), vaya encaminada a plantear la instalación de una "historia soñada", como estrategia de resistencia y transformación ante la tradicionalidad, la marginación y la ausencia de la diversidad tanto territorial como de las personas que componen el continente.

América Latina se debate entre refrenar el avance neocolonial de dominación política y cultural europea, y reafirmar las identidades culturales, los proyectos históricos y políticos que entre la unidad y la diferencia conforman la realidad del continente. El continente se reviste de un contenido utópico, un concepto de expectativa en cuanto propuesta de futuro (Kosellec, 2004). Resuenan las palabras de Torres Caicedo "habrá un momento para todos nosotros en el que no seremos ni Peruanos, ni Bolivianos, ni Argentinos, ni Dominicanos, ni Haitianos, ni Uruguayos, ni Venezolanos, ni Ecuatorianos, ni Colombianos, ni Centroamericanos, seremos todos latino-americanos" (Rojas, 2016, p. 203). 


\section{Referencias}

Apple, M. (1991). Ideología y currículo. Akal.

Apple, M. y Beane, J. (2012). Escuelas democráticas. Morata.

Arpini, A. (2013). América Latina-nuestra América. El quehacer filosófico entre nosotros. Letras, 84, 143-172.

Barton, K. (2002). Masculinity and schooling. Theory and Research in Social Education, 30(2), 306-312.

Bellei, C. (2001). ¿Ha tenido impacto la reforma educacional chilena? Proyecto Alcance y Resultados de las Reformas Educativas en Argentina, Chile y Uruguay. Mineduc.

Bonilla, A. y Martínez, I. (1992). Análisis del currículo oculto de los modelos sexistas. En M. Moreno (ed.), Del silencio a la palabra: Coeducación y reforma educativa (pp. 60-92). Ministerio de Asuntos Sociales, Instituto de la Mujer, Colección Estudios.

Cassany, D. (2006). Tras las líneas. Anagrama.

Castro, G. (2014). La historia nacional cuestionada: Interculturalidad para una enseñanza de la historia en clave latinoamericana. Contextos, 32, 47-58. https://dialnet.unirioja.es/descarga/articulo/5427164.pdf

Colegio de Profesores de Chile. (2009). Conclusiones Congreso Nacional de Educación. Docencia, 39, 4-11.

Cox, C. (2003). Reforma educativa: Resultados y desafíos de su etapa presente. Documento Mineduc, Unidad de Currículum y Evaluación UCE, 1, 15-35.

Cuesta, R. (1998). Clío en las aulas: La enseñanza de la historia en España entre reformas, ilusiones y rutinas. Akal.

Falabella, A. (2015) El mercado escolar en Chile y el surgimiento de la nueva gestión pública: El tejido de la política entre la dictadura neoliberal y los gobiernos de la centroizquierda (1979 a 2009). Revista Educación y Sociedad, 36(132), 699-79.

Fernández, E. (2012). El humanismo latinoamericanista de Arturo Andrés Roig. Revista Internacional de Filosofía Iberoamericana y Teoría social, 59, 11-26.

Foucault, M. (1979). Microfísica del poder. La Piqueta.

Fraser, N. (2008). La justicia social en la era de la política de identidad: Redistribución, reconocimiento y participación. Revista de Trabajo, 6(4), 83-99. http://trabajo.gob.ar/downloads/igualdad/08ago-dic_fraser.pdf

Giroux, H. (1990). Los profesores como intelectuales: hacia una pedagogía crítica del aprendizaje. Paidós

Giroux, H. (1998). Las políticas de educación y cultura. En H. Giroux y P. McLaren (eds.), Sociedad, cultura y educación (pp. 79-86). Niño y Dávila Editores. 
Giroux, H. (2003). Pedagogía y política de la esperanza: Teoría, cultura y enseñanza. Amorrortu Editores.

Koselleck, R. (2004). Historia de los conceptos y conceptos de historia. Revista Ayer. Asociación de Historia Contemporánea, 53, 27-45.

Ley 18.962. (1990, 10 de marzo). Ley Orgánica Constitucional de Enseñanza o LOCE. Fija los requisitos mínimos que deberán cumplir los niveles de enseñanza básica y enseñanza media.

Marolla, J. (2014). ¿Aún son invisibles las mujeres? Análisis de la presencia de la historia de las mujeres en los libros texto de secundaria chilenos. En J. Pagès y A. Santisteban (Eds.), Una mirada al pasado y un proyecto de futuro, investigación e innovación en didáctica de las ciencias sociales (pp. 305-314). Servei de Publicacions de la Universitat Autònoma de Barcelona

Marolla, J. (2019a). La inclusión de las mujeres en las clases de historia: Posibilidades y limitaciones desde las concepciones de los estudiantes chilenos. Revista Colombiana de Educación, 78, 1-24. https://doi. org/10.17227/rce.num77-6549

Marolla, J. (2019b). La didáctica de las ciencias sociales y el problema de la ausencia de las mujeres y su historia: Reflexiones en torno a un estudio de casos para transformar las prácticas de enseñanza. Revista Electrónica Educare, 23(1), 1-24.

Marolla, J. y Pagès, J. (2018). La historia reciente en los currículos escolares de Argentina, Chile y Colombia. Desafíos de la educación para la ciudadanía desde la didáctica de las ciencias sociales. Historia y Memoria, 17, 153-183. https://doi.org/10.19053/20275137.n17.2018.7455

Marolla, J. y Saavedra, C. (2020). Presencia y ausencia de América Latina: La didáctica de las ciencias sociales y la interculturalidad como desafío para la enseñanza. Revista Interdisciplinaria de Estudios Latinoamericanos, 4(1), 75-92.

Mignolo, W. (2007). La idea de América Latina: La herida colonial y la opción decolonial. Gedisa.

Ministerio de Educación República de Chile. (2009). Objetivos fundamentales y contenidos mínimos obligatorios de la Educación Básica y Media.

Ministerio de Educación República de Chile (2011a). Historia, Geografía y Ciencias Sociales. Programa de estudios primer año medio.

Ministerio de Educación República de Chile (2011b). Historia, Geografía y Ciencias Sociales. Programa de estudios segundo año medio.

Ministerio de Educación República de Chile (2015a). Historia, Geografía y Ciencias Sociales. Programa de estudios tercer año medio.

Ministerio de Educación República de Chile (2015b). Historia, Geografía y Ciencias Sociales. Programa de estudios cuarto año medio. 
Miralles, P. y Gómez, C. (2017). Enseñanza de la historia, análisis de libros de texto y construcción de identidades colectivas. Historia y Memoria de la Educación, 6, 9-28. https://doi.org/10.5944/hme.6.2017.18745

Oficina Internacional de Educación de la Unesco. (2018). Estado, mercado y currículum escolar: La experiencia chilena (1964-2018). Unesco.

Oficina Internacional de Educación Unesco. (2004). La educación chilena en el cambio de siglo: Políticas, resultados y desafíos. Unesco.

Ortega, D. y Pagès, J. (2017). Literacidad crítica, invisibilidad social y género en la formación del profesorado de Educación Primaria. Reidics, Revista de Investigación en Didáctica de las Ciencias Sociales, 1, 102117. https://doi.org/10.17398/2531-0968.01.118

Ortega, D., Marolla, J. y Heras, D. (2020). Invisibilidades sociales, identidades de género y competencia narrativa en los discursos históricos del alumnado de educación primaria. En E. J. Díez Gutiérrez; J. R. Rodríguez Fernández, Educación para el bien común: Hacia una práctica crítica, inclusiva y comprometida socialmente (pp. 89-104). Octaedro.

Pagès, J. (1999). Las representaciones previas de los estudiantes de maestro de ciencias sociales, geografía e historia. Teoría y Didáctica de las Ciencias Sociales, 4, 161-178.

Pagès, J. y Mariotto, O. (2014). Los actores invisibles de la historia: Un estudio de caso de Brasil y Cataluña. En J. Pagès y A. Santisteban (eds.), Una mirada al pasado y un proyecto de futuro: Investigación en didáctica de las ciencias sociales (pp. 37-44). AuPDCs/Servei de Publicacions de la Universitat Autònoma de Barcelona.

Pagés, J. y Sant, E. (2012). Las mujeres en la enseñanza de la Historia: ¿Hasta cuándo serán invisibles? Cadernos de Pesquisa do cDhis, 25(1), 91-117.

Pagès, J. y Santisteban, A. (2011). Les qüestions socialment vives I I'ensenyament de les ciències socials. Servei de Publicacions de la Universitat Autònoma de Barcelona

Pinar, W. (2014). La teoría del currículum. Narcea.

Pinochet, S. (2014). La enseñanza de la historia de la infancia como una herramienta para potenciar la formación ciudadana y democrática en la escuela. En J. Pagès y A. Santisteban (eds.), Una mirada al pasado y un proyecto de futuro, investigación e innovación en didáctica de las ciencias sociales (pp. 715-724). Servei de Publicacions de la Universitat Autònoma de Barcelona.

Pinochet, S. (2015). Mirándonos al espejo: Los estudiantes frente a los niños, niñas y jóvenes como protagonistas de la Historia. En A. Hernández, C. García y J. L. de la Montaña (Eds.), Una enseñanza de las ciencias sociales para el futuro: Recursos para trabajar la invisibilidad de personas, lugares y temáticas (pp. 967-976). Universidad de Extremadura y AUPDCS. 
Plá, S. (2016). Currículo, historia y justicia social: Estudio comparativo en América Latina. Revista Colombiana de Educación, 71, 53-77. http:// www.scielo.org.co/pdf/rcde/n71/n71a03.pdf

Quijano, A. (2000). Colonialidad del poder, eurocentrismo y América Latina. En E. Lander (ed.), La colonialidad del saber: Eurocentrismo y ciencias sociales (pp. 201-246) Clacso.

Roig, A. (1993). Historia de las ideas teoría del discurso y pensamiento latinoamericano. Editorial Universidad de Santo Tomás.

Rojas, D. (2016). Los latinoamericanos de París en el cambio de siglo. Colombia Internacional, 87, 243-259. https://doi.org/10.7440/colom biaint87.2016.10

Saladino, A. (2010). El latinoamericanismo como pensamiento decolonizador. Revista Universum, 25(2), 179-186.

Santisteban, A. (2015). La formación del profesorado para hacer visible lo invisible. En A. Hernández, C. García y J. L. de la Montaña (Eds.), Una enseñanza de las ciencias sociales para el futuro: Recursos para trabajar la invisibilidad de personas, lugares y temáticas (pp. 383393). Universidad de Extremadura. Asociación Universitaria del Profesorado de Didáctica de las Ciencias Sociales.

Santos, B. (2009). Refundación del Estado en América Latina. IIDS.

Santos, B. (2011). Epistemologías del Sur. Utopía y Praxis Latinoamericana, 16(54), 17-39.

Santos, B. (2014). Derechos humanos, democracia y desarrollo. Colección de Justicia.

Santos, B. (2017). Justicia entre saberes: Epistemologías del Sur contra el epistemicidio. Morata.

Sarlo, B. (2012) Tiempo pasado. Cultura de la memoria y giro subjetivo una discusión. Siglo xxı.

Spivak, G. (2012). Feminism and critical theory. En G. Spivak (Eds.), In other worlds: Essays in cultural politics (pp. 55-70). Routledge.

Tamayo, J. (1975). Geografía de América. Fondo de Cultura Económica.

Torres, L. (2016). Historia reciente en la escuela colombiana: Acercamiento a las nociones de memoria, historia y conflicto. Revista Colombiana de Educación, 71, 165-185. http://www.scielo.org.co/ $\mathrm{pdf} / \mathrm{rcde} / \mathrm{n} 71 / \mathrm{n} 71 \mathrm{a} 07 . \mathrm{pdf}$

Tosar, B. (2018). Literacidad crítica y enseñanza de las ciencias sociales en primaria: Profe, las bolsas de plástico no son medusas. Reidicis, Revista de Investigación en Didáctica de las Ciencias Sociales, 2, 4-19. https://doi.org/10.17398/2531-0968.02.4

Tubino, F. (2005). Entre el multiculturalismo y la interculturalidad: más allá de la discriminación positiva. En N. Fuller (Ed.), Interculturalidad y política: Desafíos y posibilidades (pp. 51-76). RDCSP. 
Walsh, C. (2012) Interculturalidad crítica y (de)colonialidad: Ensayos desde Abya Yala. Abya-Yala.

Woyshner, C. (2002). Political history as women's history: Toward a more inclusive curriculum. Theory and Research in Social Education, 30(3), 354-380. 10.1080/00933104.2002.10473201

Zea, L. (1971). Latinoamérica, emancipación y neoloconialismo. Tiempo Nuevo. 